\title{
GABA-Mediated Inactivation of Medial Prefrontal and Agranular Insular Cortex in the Rat: Contrasting Effects on Hunger- and Palatability-Driven Feeding
}

\author{
Brian A Baldo*, ${ }^{*, 2}$, Robert C Spencer ${ }^{3}$, Ken Sadeghian' and Jesus D Mena ${ }^{2}$ \\ 'Department of Psychiatry, School of Medicine and Public Health, University of Wisconsin-Madison, Madison, WI, USA; ${ }^{2}$ Neuroscience Training \\ Program, School of Medicine and Public Health, University of Wisconsin-Madison, Madison, WI, USA; ${ }^{3}$ Department of Psychology, Graduate School, \\ University of Wisconsin-Madison, Madison, WI, USA
}

\begin{abstract}
A microanalysis of hunger-driven and palatability-driven feeding was carried out after muscimol-mediated inactivation of two frontal regions in rats, the agranular/dysgranular insular cortex (AIC) and the ventromedial prefrontal cortex (vmPFC). Food and water intake, feeding microstructure, and general motor activity were measured under two motivational conditions: food-deprived rats given standard chow or ad libitum-fed rats given a palatable chocolate shake. Muscimol infusions into the AIC diminished intake, total feeding duration, and average feeding bout duration for the palatable-food condition only but failed to alter exploratory-like behavior (ambulation or rearing). In contrast, intra-vmPFC muscimol infusions did not alter the overall intake of chow or chocolate shake. However, these infusions markedly increased mean feeding bout duration for both food types and produced a modest but significant reduction of exploratory-like behavior. The lengthening of feeding-bout duration and reduction in rearing were mimicked by intra-vmPFC blockade of AMPA-type but not NMDA-type glutamate receptors. Neither water consumption nor the microstructure of water drinking was affected by inactivation of either site. These results indicate a regional heterogeneity in frontal control of feeding behavior. Neural processing in AIC supports palatability-driven feeding but is not necessary for intake of a standard food under a food-restriction condition, whereas ventromedial prefrontal cortex, and AMPA signaling therein, modulates the duration of individual feeding bouts regardless of motivational context. Results are discussed in the context of regionally heterogeneous frontal modulation of two distinct components of feeding behavior: reward valuation based upon taste perception (AIC) vs switching between ingestive and non-ingestive (eg, exploratory-like) behavioral repertoires (vmPFC).

Neuropsychopharmacology (20I6) 4I, 960-970; doi:I 0.I 038/npp.2015.222; published online 2 September 2015
\end{abstract}

\section{INTRODUCTION}

Evidence suggests that the non-homeostatic intake of palatable, energy-dense foods contributes to the development of obesity (Morris et al, 2014). Although the neural circuitry governing non-homeostatic eating is not fully understood, one hypothesized route of control involves the 'hijacking' of energy balance-regulatory circuits by telencephalic projections conveying information related to food motivation and cognition (Berthoud, 2011). Recently, there has been considerable interest in identifying such 'top-down' pathways originating in frontal cortex (Petrovich et al, 2005; Mena et al, 2013; Land et al, 2014). A network of sites in the insular, orbitofrontal, and medial-prefrontal cortical regions participate in several processes relevant to non-homeostatic eating; for example, computing representations of the

* Correspondence: Dr BA Baldo, Department of Psychiatry, University of Wisconsin-Madison, School of Medicine and Public Health, 600I Research Park Boulevard, Madison, WI 53719, USA, Tel: +| 608263 4019, Fax: + 608265 3050, E-mail: babaldo@wisc.edu

Received 15 April 2015; revised 21 May 20I5; accepted 10 June 20I5; accepted article preview online 23 July 2015 stimulus properties and reward value of palatable food, and modulating food-directed consummatory actions (Small et al, 2001; Rolls, 2012; Horst and Laubach, 2013; Jezzini et al, 2013). Human imaging studies have revealed that the aforementioned areas are strongly activated by cues depicting palatable, calorie-dense foods, and these responses are exaggerated in obese individuals (Killgore et al, 2003; Goldstone et al, 2009; Martin et al, 2010). Hence, there may be select frontal cortical sites that contribute disproportionately (or, perhaps, selectively) to non-homeostatic palatable feeding relative to normal homeostatic intake engendered by energy deficit. Identifying such sites is, however, complicated by the fact that palatability and food-reward valuation are enhanced in energy-deficit states (Cabanac and Duclaux, 1973; Berridge, 1991; Cameron et al, 2014).

One way to disentangle these issues would be to compare the effects of manipulating a given site on the intake of standard food during acute energy deficit $v s$ intake of a highly palatable food under no energy deficit. We used this approach to explore the potentially dissociable roles of the ventral medial prefrontal cortex (vmPFC) and the agranular/dysgranular region of insular cortex (AIC) in palatability-driven $v s$ energy deficit-driven feeding. Both the vmPFC and AIC 
have been identified as highly responsive to food cues in human imaging studies, and both are anatomically well positioned to modulate feeding behavior, for the following reasons. On the input side, areas within insular cortex represent primary gustatory cortex in human and nonhuman primates and receive abundant convergent somatosensory, viscero-sensory, olfactory, and limbic input relevant to feeding (Pritchard et al, 1986; Yaxley et al, 1990; Small, 2010). The rodent homolog of these insular areas is located in the AIC (Kosar et al, 1986; Allen et al, 1991; Maffei et al, 2012). Similarly, the vmPFC receives highly processed gustatory and olfactory information along with limbic input from the amygdala and hippocampus (Petrovich et al, 1996; Shi and Cassell, 1998; Gabbott et al, 2003). On the output side, both the vmPFC and AIC send direct projections to feedingmodulatory hypothalamic regions (Hurley et al, 1991; Floyd et al, 2001). Additionally, vmPFC (and, to a lesser extent, the AIC) sends strong projections to the nucleus accumbens shell (Vertes, 2004; Reynolds and Zahm, 2005), a site at which profound hyperphagia is elicited by GABA or opiate receptor stimulation (Basso and Kelley, 1999; Newman et al, 2013).

In the present study, intra-site infusions of the GABA-A receptor agonist, muscimol, were used to produce 'temporary lesions' in the vmPFC and AIC of rats. Ingestive behavior and associated motor activity were then recorded in two motivational situations: food-deprived rats presented with standard chow, and ad libitum-fed rats presented with a palatable high sugar/fat chocolate-flavored shake. The overarching goal was to compare the effects of muscimol inactivation across feeding conditions and across sites, in order to ascertain whether either site has a selective role in modulating the expression of non-homeostatic, palatabilitydriven feeding.

\section{MATERIALS AND METHODS}

All procedures were evaluated and approved by the Institutional Animal Care and Use Committee of the University of Wisconsin-Madison and are in accordance with the guidelines stated in the NIH Guide for the Care and Use of Laboratory Animals. Facilities have been approved by the American Association for the Accreditation of Laboratory Animal Care.

\section{Subjects}

Male Sprague-Dawley rats, obtained from Harlan (Indianapolis, IN), weighing 275-290 g upon arrival in the laboratory, were used in all experiments. Rats were pairhoused in clear polycarbonate cages (9.5 inches width $\times$ 17 inches length $\times 8$ inches height), with cob bedding, in a light- and temperature-controlled vivarium. Animals were maintained under a 12:12-h light/dark cycle (lights on at 0700 hours). Food (Agway rat-chow pellets) and water were available ad libitum, except during food-restriction phases of the experiments as described in the Experimental Design section. Animals were handled gently daily to reduce stress.

\section{Surgical Procedures}

Rats (weighing 330-370 g at the time of surgery) were anesthetized with a xylazine/ketamine mixture $(13 \mathrm{mg} / \mathrm{kg}$ xylazine plus $87 \mathrm{mg} / \mathrm{kg}$ ketamine, i.p.) and secured in a Kopf stereotaxic frame. The toothbar was set at $-4.0 \mathrm{~mm}$ below the interaural line. Bilateral stainless-steel cannulas $(10 \mathrm{~mm}$ long, 23 gauge) were implanted using standard stereotaxic procedures (see Perry et al, 2009). For the vmPFC placements, cannulas were placed at an acute angle to the midline to avoid damaging the medial wall of the cortex. For the vmPFC, coordinates of the injection site were AP: $+3.0 \mathrm{~mm}$ from bregma, ML: $2.0 \mathrm{~mm}$ from midline, DV: $-5.2 \mathrm{~mm}$ from skull surface with cannulas angled at 19 degrees from vertical. For the AIC, coordinates were AP: $+1.3 \mathrm{~mm}$ from bregma, ML: $4.1 \mathrm{~mm}$ from midline, and DV: $-6.5 \mathrm{~mm}$ from skull surface (angled placements not used). Cannulas were fixed in place $2.5 \mathrm{~mm}$ above the target sites with dental acrylic (New Truliner, Skokie, IL) and anchoring skull screws (Plastics One, Roanoke, VA). Wire stylets (10 mm long, 30 gauge) were placed in the cannulas to prevent blockage. Rats were given an intramuscular injection of Penicillin $(0.3 \mathrm{ml}$ of a $300000 \mathrm{unit} / \mu \mathrm{l}$ suspension; Phoenix Pharmaceuticals. St Joseph, MO), placed in warm recovery cages, returned to their home cages upon awakening, and given a recovery period of no less than 5 days (with daily health checks and handling).

\section{Drugs and Microinfusions}

Muscimol, CNQX disodium salt, and AP-5 were all obtained from Sigma-Aldrich. Drugs were dissolved in sterile $0.9 \%$ saline. For CNQX, saline was warmed slightly during drug preparation to aid solubility. For intracerebral microinfusions, rats were held gently, injectors were lowered through the guide cannulae, and infusions were made at a rate of $0.32 \mathrm{ml} /$ min. Additional details are given in Supplementary Materials.

\section{Behavioral Testing Procedures}

Behavioral testing was carried out in clear polycarbonate behavior-observation cages (9.5 inches width $\times 17$ inches length $\times 8$ inches height) with wire-grid floors. Experiments were conducted under two conditions: (1) a food-deprivation condition in which pre-weighed quantities of chow pellets were offered in the testing cage after $20 \mathrm{~h}$ of food deprivation or (2) a palatable-feeding condition in which liquid chocolate Ensure Plus Milk Chocolate flavor (hereafter referred to as 'chocolate shake') was offered in the ad libitum state. This shake consisted of maltodextrin, sucrose, milk and soy protein, corn and canola oils, vitamins, and natural plus artificial chocolate flavor (\% kcal/serving: fat, 29; carbohydrate, 57; protein, 14). Chocolate shake was available from an overhead graduated buret in the testing cage. Water was available in all testing conditions from an overhead graduated buret. Rats were rated by an experimenter blind to treatment, using an event recorder interfaced to a PC computer. The following behaviors were monitored:

Cage crossings: ambulation across the midpoint of the cage's long axis,

Rears: rearing up on the hind legs,

Feeding: uninterrupted bouts of chow-pellet or chocolateshake intake, and

Drinking: uninterrupted bouts of water intake. 
The frequency, duration, and latency to initiate all these behaviors were recorded, except for cage crossings, for which only frequency was recorded. To record the duration of a given behavioral event, a switch was depressed that started an automatic timer (specific for that behavior) at the initiation of the behavior. The timer was switched off when the behavior was interrupted by a different behavior (for example, a rat engaged in a drinking bout stopping his drinking to rear or ambulate across the cage). From the frequency and duration data, the overall mean duration of each rearing, feeding, or drinking bout was calculated.

\section{Experimental Design}

Two separate groups of rats were used, with infusion cannulae aimed at either the $\operatorname{vmPFC}(n=8$ rats $)$ or AIC ( $n=8$ rats). After recovery from surgery, all rats were given 'sham injections' in which injection tubing was lowered into the infusion cannulae, but not into the brain, in order to acclimate the rats to handling and infusion.

On the evening of the sham injection day, rats were placed into either a food-deprivation cohort or a chocolate-shake cohort. Specifically, half the rats in each brain site group were placed on a food-restriction schedule in which food was taken away overnight, $20 \mathrm{~h}$ before the next day's behaviorobservation session. These rats were allowed to eat ad libitum immediately after each behavior-observation session and over the interim day or days that separated sessions, whereupon food was again removed overnight before the next testing session. This feeding schedule was maintained throughout the experiment. The other half of each brain site group was given bottles containing chocolate shake (in addition to their usual chow) in their home cages overnight for one night only. This served to accommodate the rats to the novel food. Thenceforth, rats received chocolate shake only during 30-min behavioral observation sessions (never in the home cages). Rats in the chocolate-shake cohort were not food deprived.

To further acclimate the rats to microinfusion and testing procedures, on the day after the first exposure to food deprivation or chocolate shake, all rats were given saline injections into their respective targeted brain sites and were placed into the behavior-observation cages with either chow pellets plus water bottles (for the food-restricted rats) or bottles of chocolate shake plus water bottles (for the ad libitum-fed rats) for $30 \mathrm{~min}$. Two days later, drug testing commenced.

On drug testing days, rats were injected with muscimol $(0,30$, or $75 \mathrm{ng})$ and placed immediately into the behaviorobservation cages. Unconditioned motor and ingestive behaviors were monitored and recorded for $30 \mathrm{~min}$ as described in Behavioral Testing Procedures section. In these sessions, rats were offered either chow and water or chocolate shake and water, depending upon their group assignment. Each rat received every dose according to within-subjects Latin square designs. Testing days were separated from each other by at least one interim day.

After completion of all treatments, the food-restriction and chocolate-shake groups were reversed, rats were given 2 days to accommodate to their new food schedules, and within-subjects testing of all drug doses was carried out a second time. Hence, all doses were tested in all rats, under both food-deprivation+chow and ad libitum+chocolateshake conditions.

A similar experimental design was used to test the effects of the AMPA-type glutamate receptor antagonist, $\mathrm{CNQX}(0,1$, $5 \mu \mathrm{g} / \mathrm{side}$ ), and the NMDA-type glutamate receptor antagonist, AP-5 $(0,0.1,1.0 \mu \mathrm{g} / \mathrm{side})$. The effects of intra-vmPFC CNQX and AP- 5 were tested in separate groups of rats $(n=8$ per group), with the order of chow and chocolate-shake presentation counterbalanced within rats as described above. Doses for both drugs were within the behaviorally active intracerebral dose ranges reported in the literature (Baldwin et al, 2000; Bishop et al, 2011; Mena et al, 2013; Tan et al, 2014).

\section{Verification of Placements}

At the end of each experiment, rats were deeply anesthetized with sodium pentobarbital and perfused transcardially with a $0.9 \%$ saline solution followed by $10 \%$ formalin in phosphate buffer. Brains were collected and stored in $10 \%$ formalin. Coronal sections $(60 \mu \mathrm{m})$ were cut through the infusion site on a cryostat microtome, collected on slides, stained with cresyl violet, and subsequently reviewed to verify correct placement of the intracortical injections. Images of representative sections from each experiment were captured using the Scion Image software on a computer interfaced with a microscope-mounted Hitachi HV-C20 CCD camera.

\section{Statistical Analyses}

For the intra-vmPFC and intra-AIG muscimol studies, data were analyzed using three-factor ANOVAs, with one between-subjects factor (brain region) and two withinsubjects factors (dose, food type). Significant $(P<0.05)$ interactions of brain region with the other two factors were further explored using two-factor (food type $\times$ dose) repeated-measures ANOVAs in each brain region separately. Means comparisons were conducted using Fisher's PLSD test, as informed by which factor was significant in the ANOVA (ie, either full means comparisons or comparisons collapsed across the non-significant factor were carried out). The intra-vmPFC AP-5 and CNQX experiment was analyzed in a similar way, ie, with three-factor ANOVAs (drug type $\times$ food type $\times$ dose) followed by two-factor ANOVAs for each drug separately, and using Fisher's PLSD tests following significance in the two-factor ANOVAs. Note that, because data for chow and chocolate-shake intake were in different units ( $\mathrm{g}$ and $\mathrm{ml}$, respectively), these measures were analyzed separately with two-factor ANOVAs (food type $\times$ dose).

\section{RESULTS \\ Histological Verification of Placements, and Accounting of Subjects Omitted from Analyses}

As shown in Figure 1, for the vmPFC, placements fell within the infralimbic zone of medial prefrontal cortex, with a significant number of placements in the dorsal portion of infralimbic cortex, impinging on the ventral border of the prelimbic area (Figure 1a). For the AIC, placements fell mainly in the dorsal aspects of dorsal AIC near its border with dysgranular cortex, with a small number of placements 
a

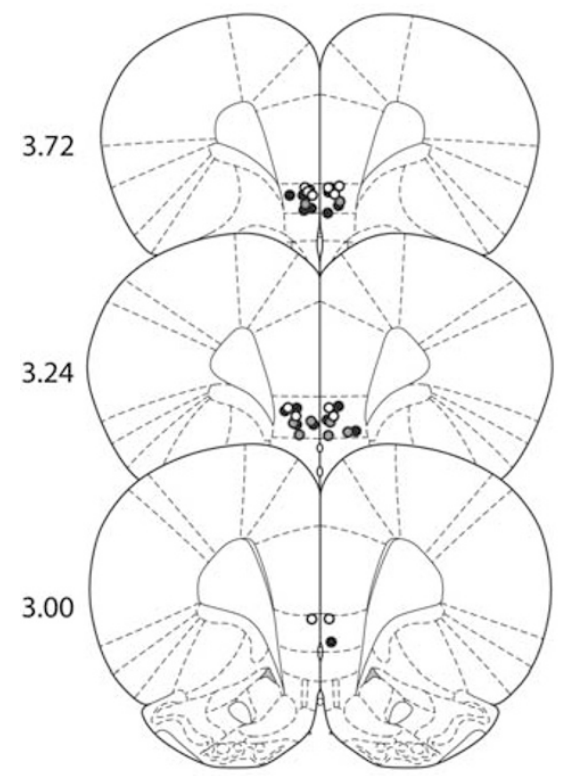

b

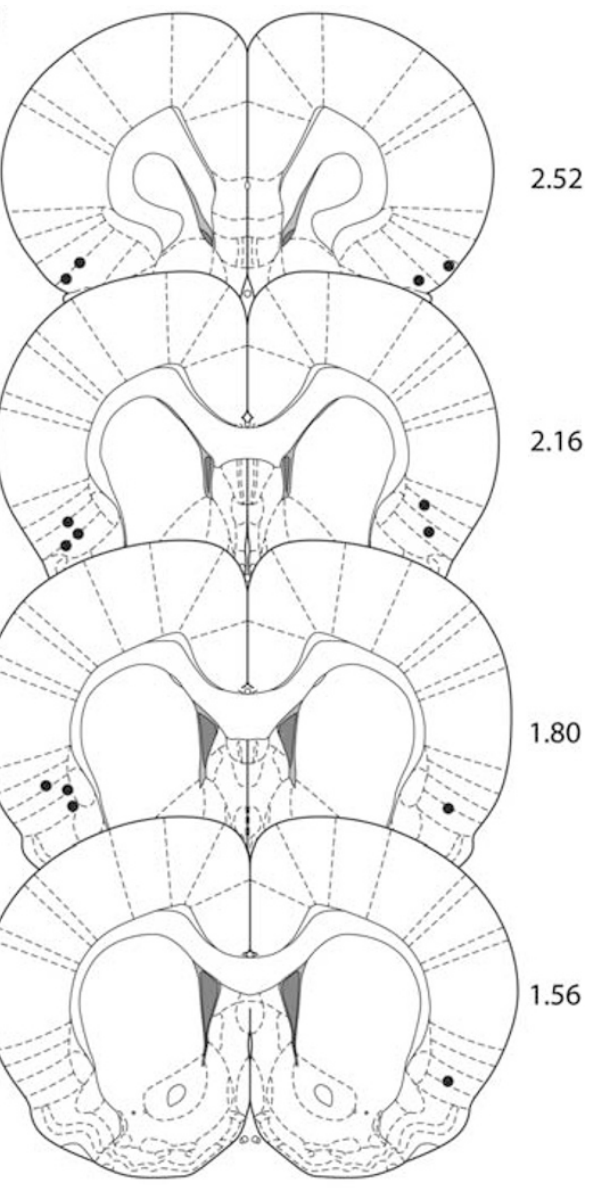

d

C

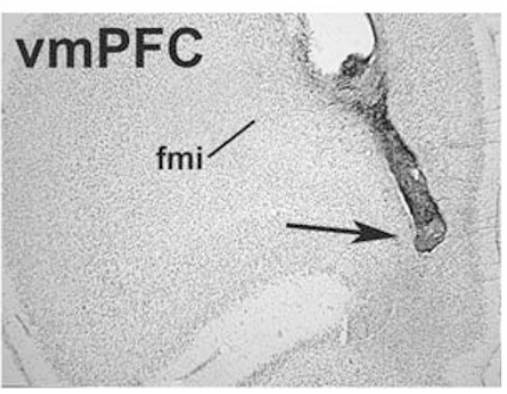

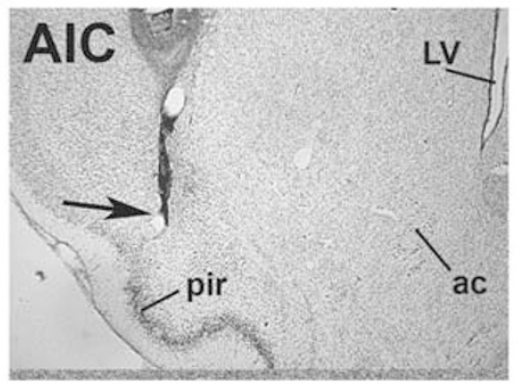

Figure I Chartings of injector-tip placements in the VmPFC (a) and AIC (b), and photomicrographs of placements within each site (c, d). (a) Black circles depict placements from the muscimol experiment, gray circles depict placements from the CNQX experiment, and white circles depict placements from the AP-5 experiment. (b) Black circles depict placements from the muscimol experiment. (c) vmPFC placement; fmi = corups callosum, anterior forceps. (d) AIC placement; pir = piriform cortex, ac = anterior commissure, $\mathrm{LV}=$ lateral ventricle. In panels (c) and (d), arrows depict injector tips.

localized in anterior ventral AIC (Figure 1b). Histological examination of injector tracks revealed no unusual damage (Figure 1c and d).

For one out of eight subjects in the muscimol/AIG study, and for two of the eight rats in the AP5/vmPFC study, bilateral placements fell outside the targeted zones; for the CNQX study, two of the eight rats became ill and were euthanized. All of these rats were dropped from the analyses.

\section{Food and Water Intake}

As shown in Figure 2, muscimol infusions into the AIC produced distinct effects on intake, compared with
intra-vmPFC infusions (brain region $\times$ dose interaction: $\mathrm{F}(2,26)=3.9, P=0.032)$. Further analyses within each brain region revealed that muscimol infusion into the AIC (but not the vmPFC) significantly depressed chocolate-shake intake $(\mathrm{F}(2,12)=18.5, P=0.0002$, Figure $2 \mathrm{~d})$, but not chow intake (Figure 2b), as confirmed by post-hoc analyses indicating significant differences between means associated with saline compared with each of the two muscimol doses for chocolate shake only. In contrast, deprivation-induced chow intake was unaffected by muscimol infusions into either brain region (Figure 2a and $b$ ).

Analysis of water intake revealed a significant main effect of food type $(F(1,13)=35.01, P<0.0001)$, reflecting the fact 
AIC
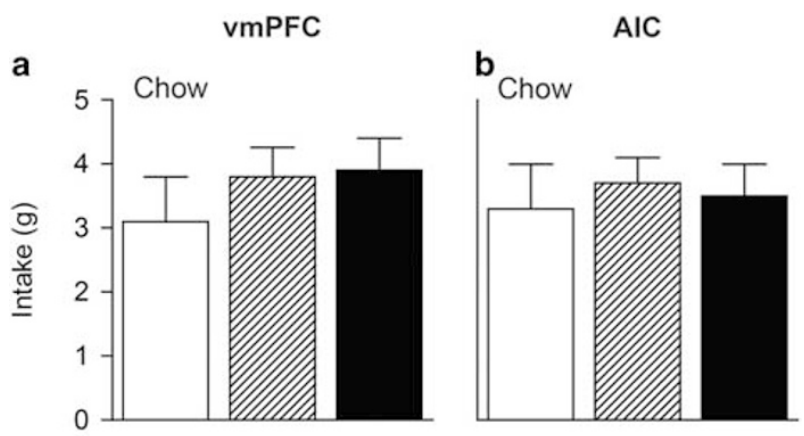

C
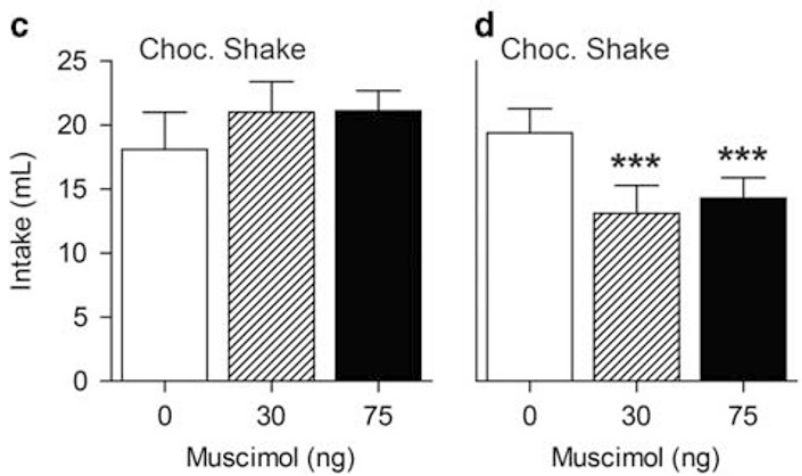

Figure 2 Intake of chow and chocolate shake ('Choc. Shake') after muscimol infusions into the ventral medial prefrontal cortex ('vmPFC'; panels a and c) or agranular insular cortex ('AIC'; panels b and d). Muscimol doses for the bilateral injections are shown in $\mathrm{ng} /$ side. Error bars depict one SEM. $* * * P<0.001$, different from corresponding vehicle treatment.

that there was more prandial water drinking in the chow condition relative to the chocolate-shake condition regardless of brain region or muscimol dose, and a significant effect of brain region $(\mathrm{F}(1,13)=6.7, P=0.023)$, reflecting the fact that overall water intake tended to be slightly higher in the vmPFC-cannulated group relative to AIC-cannulated group, regardless of muscimol dose. These data are shown in Supplementary Table S1.

\section{Microanalysis of Feeding}

Muscimol infusions produced distinct effects on feeding microstructure in the vmPFC $v s$ the AIC (Figure 3). Analysis of total eating duration per session revealed a significant main effect of food type $(\mathrm{F}(1,13)=11.7, P=0.0046)$, a food type $\times$ brain region interaction $(\mathrm{F}(1,13)=10.0, P=0.0074)$, and a food type $\times$ dose interaction $(\mathrm{F}(2,26)=5.6, P=0.0095)$, and analysis of the average duration of eating bouts per session showed a significant main effect of brain region $(\mathrm{F}(1,13)=6.4, P=0.025)$, a food type $\times$ brain region interaction $(\mathrm{F}(1,13)=7.4, \quad P=0.018)$, and a dose $\times$ brain region interaction $(\mathrm{F}(2,26)=15.9, \quad P<0.0001)$. These multiple interactions were followed by two-factor ANOVAs (dose $x$ food type) on data for each brain region separately, as described below.

\section{Muscimol Effects in the AIC}

Total feeding duration for chocolate shake (but not chow) was markedly suppressed by intra-AIC muscimol (main effect of food type: $\mathrm{F}(1,6)=13.2, P=0.01$; dose $\times$ food type interaction: $F(2,12)=5.5, P=0.02$; Figure $3 \mathrm{~d}$ ). Dose-wise comparisons indicated that, for chocolate-shake intake, 30-ng muscimol produced a significant decrease in total duration relative to saline, whereas the effect of $75 \mathrm{ng}$ muscimol approached but did not achieve statistical significance $(P=0.08)$. Mean eating bout duration was also reduced by muscimol, again, more strongly in the chocolate-shake condition (Figure 3f). There was a main effect of food type $(F(1,6)=19.0, P=0.0048)$ and dose $(\mathrm{F}(2,12)=3.7, P=0.05)$, but no interaction. Post-hoc tests indicated that mean bout durations (collapsed across the dose factor) were lower in the chocolate-shake $v s$ chow condition and that mean bout durations (collapsed across the food-type factor) for each of the two muscimol doses were lower than saline. These effects on eating duration and bout size are in accordance with the decrease in chocolateshake intake produced by AIC inactivation (see above). Muscimol produced no effects on eating duration in the chow condition. Note that, overall, rats initiated a greater number of feeding bouts in the chocolate-shake condition (main effect of food type: $\mathrm{F}(1,6)=10.4, \quad P=0.018$; Figure $3 \mathrm{~b}$ ). Together, these data suggest that intra-AIC muscimol diminished palatable feeding by shifting the microstructure of chocolate-shake ingestion toward numerous, unusually brief, feeding bouts. Finally, no effects were noted with regard to the latency to initiate feeding, either in the chow or chocolate-shake condition (Supplementary Table S2).

\section{Muscimol Effects in the vmPFC}

In contrast to effects seen in the AIC, intra-vmPFC muscimol markedly increased the mean duration of each feeding bout per session in each food-type condition (main effect of dose: $\mathrm{F}(2,14)=13.6, P=0.0005$; Figure 3e). There was no main effect of food type or dose $\times$ food type interaction. Post-hoc tests on muscimol dose collapsed across the food-type factor confirmed that this effect was due to a marked increase in mean feeding bout duration at each of the two muscimol doses, relative to saline treatment. The number of feeding bouts initiated, total duration of feeding, and latency to initiate the first feeding bout were not affected by intra-vmPFC muscimol (Figure 3 and Supplementary Table S2).

\section{Effects of CNQX and AP-5 in the vmPFC}

To further study the neuropharmacological basis of the subtle but significant change in feeding microstructure observed in the vmPFC, the effects of intra-vmPFC infusions of CNQX or AP- 5 on chow and chocolate-shake intake were explored in this brain site, using separate groups for each drug. Neither drug significantly altered chow or chocolateshake intake (Figure 4a and b). Nevertheless, the two drugs produced distinguishable effects on feeding microstructure, with CNQX recapitulating key effects of intra-vmPFC muscimol infusions. There were main effects of drug-type for the number of eating bouts per session $(\mathrm{F}(1,10)=5.5$, $P=0.04)$, total eating duration per session $(F(1,10)=21.7$, $P=0.0009)$, and mean eating bout duration per session $(\mathrm{F}(1,10)=19.4, \quad P=0.0013)$. Furthermore, there were drug $\times$ dose interactions for the number of eating bouts $(\mathrm{F}(2,20)=5.9, P=0.0097)$ and mean eating bout duration 
vmPFC

a

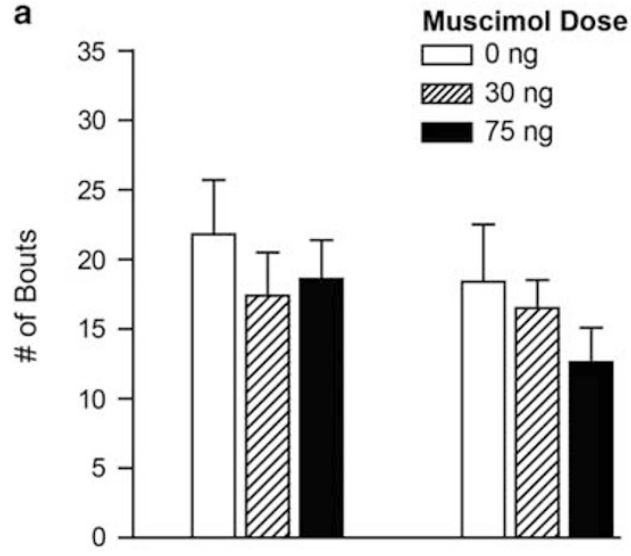

c

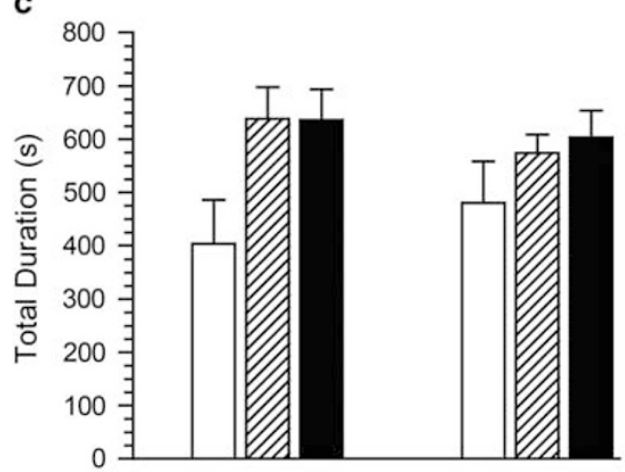

e

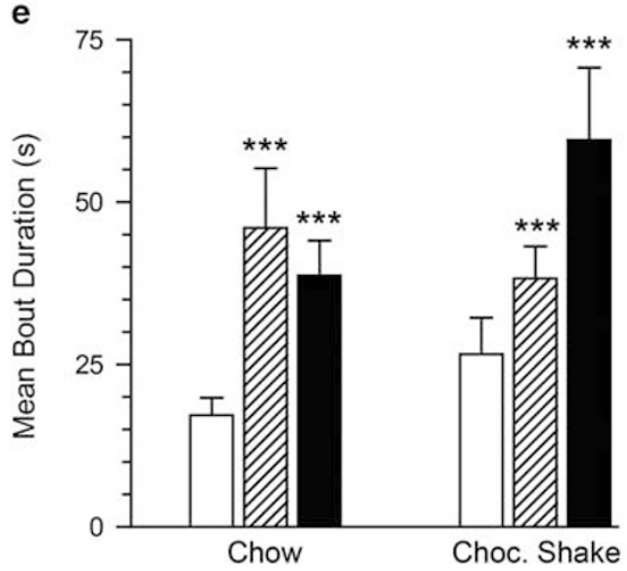

AIC

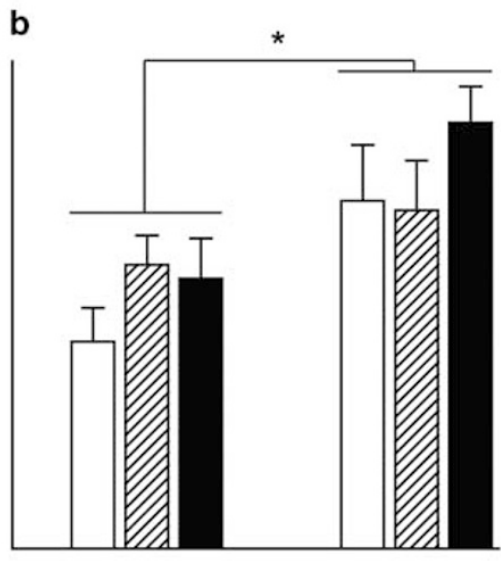

$\star *$

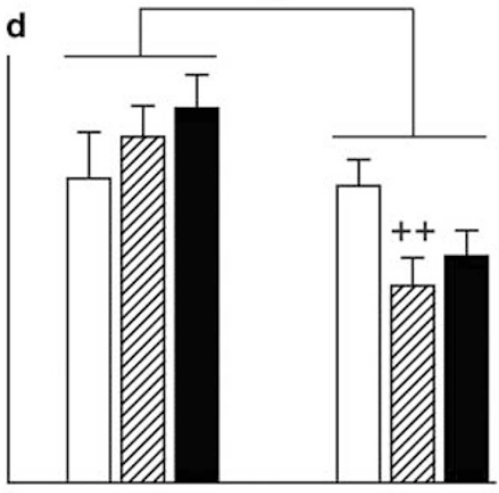

f

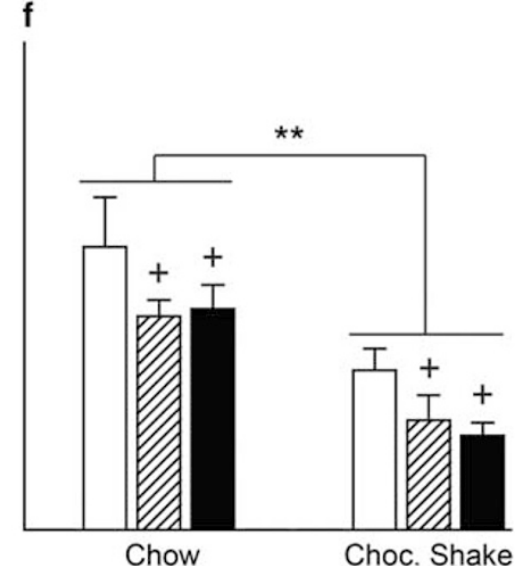

Figure 3 Microanalysis of chow intake in food-deprived rats, and chocolate-shake intake in ad libitum-maintained rats, after muscimol infusions into the ventral medial prefrontal cortex (vmPFC) or agranular insular cortex (AIC). Reported here are the number (\#) of bouts initiated per 30-min session (panels $\mathrm{a}$ and $\mathrm{b}$ ), the total duration of feeding per session (panels $\mathrm{c}$ and $\mathrm{d}$ ), and the mean bout duration for feeding per session (panels e and $\mathrm{f}$ ). Muscimol doses for the bilateral injections are shown in ng/side. (b) $* P<0.05$, main effect of food type. (d) ${ }^{*} * P<0.01$, main effect of food type; ${ }^{++} P<0.0$ I, different from vehicle chocolate-shake condition. (e) $* * * P<0.00 \mathrm{I}$, different from vehicle, when collapsed across the food-type factor (following a main effect of dose). (f) ${ }^{*} * P<0.01$, main effect of food type; ${ }^{+} P<0.05$, when collapsed across the food-type factor (following a main effect of dose). Error bars depict one SEM.

$(\mathrm{F}(2,20)=5.8, P=0.01)$, a food type $\times$ dose interaction for number of eating bouts $(\mathrm{F}(2,20)=3.7, P=0.045)$, and a drug $\times$ food type interaction for total eating duration per session $(\mathrm{F}(1,10)=7.1, P=0.024)$. These multiple main effects and interactions were followed by two-factor ANOVAs (food-type $\times$ dose) for each drug separately. These analyses indicated that CNQX significantly diminished the number of eating bouts while markedly increasing the mean duration of feeding bouts (main effect of dose for eating bouts: $\mathrm{F}(2,10)=16.2, P=0.0007$; Figure $4 \mathrm{c}$ ), for mean bout duration: $(\mathrm{F}(2,10)=6.8, P=0.014$; Figure $4 \mathrm{~g})$. These effects were observed equivalently for both food types, as indicated by the lack of food type $\times$ dose interactions. There were no effects of CNQX on total eating duration per session, and there were no effects of intra-vmPFC AP-5 for any of these measures (Figure 4). 
CNQX

a

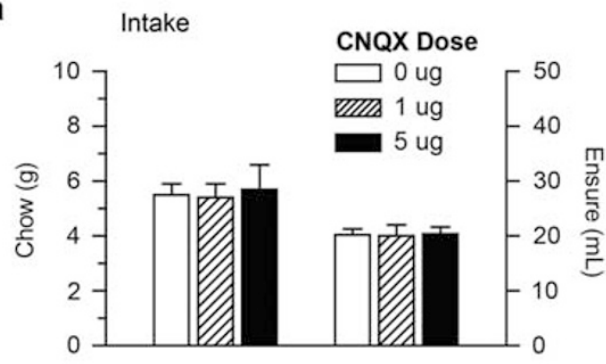

C

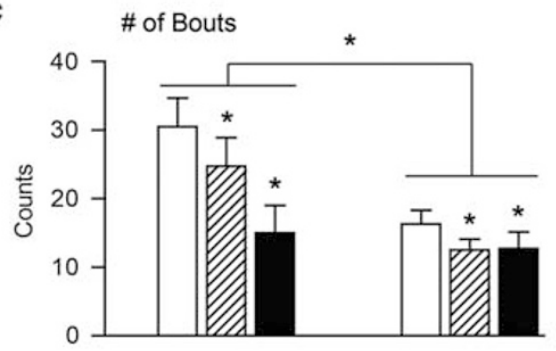

e

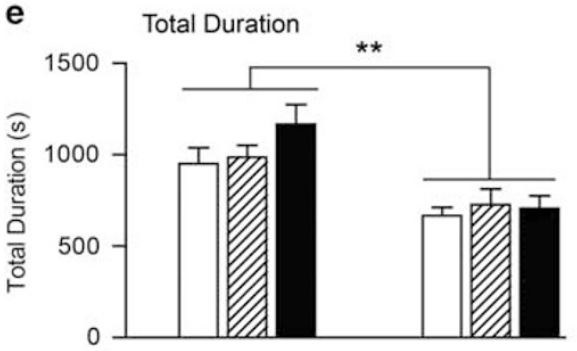

g

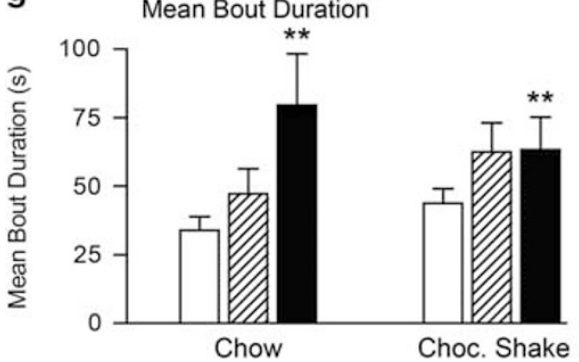

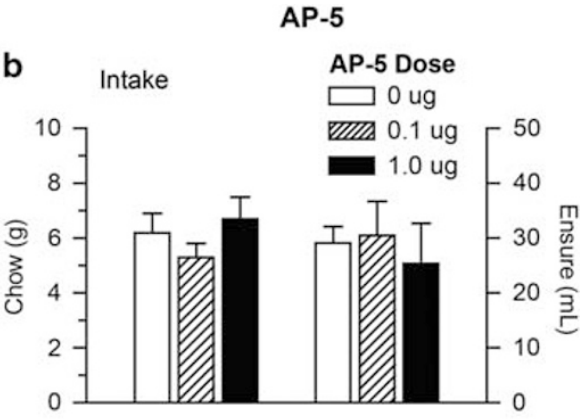

d

\# of Bouts

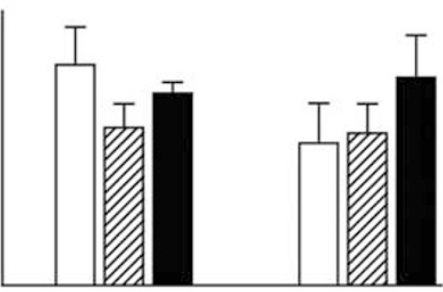

f

Total Duration

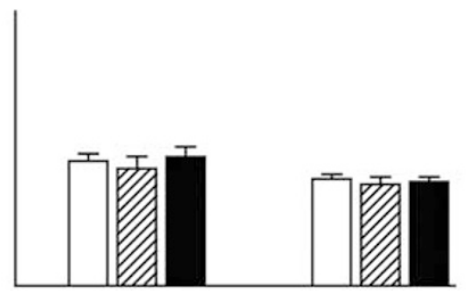

h

Mean Bout Duration

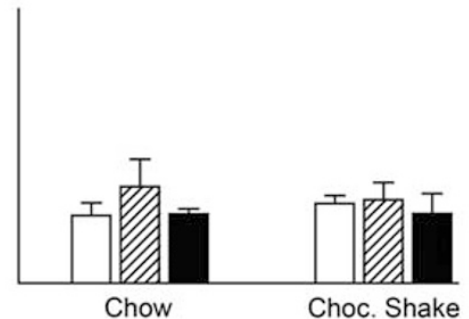

Figure 4 Chow intake, chocolate-shake intake, and microanalysis of chow and chocolate-shake intake after intra-vmPFC infusions of the AMPA/kainate-type glutamate receptor antagonist, CNQX (panels a, c, e, and g), or the NMDA-type glutamate receptor antagonist, AP-5 (panels b, d, f, and h). Axis labels for microstructure measures are the same as described in the legend for Figure 3. Doses for the bilateral drug injections are shown in $\mu \mathrm{g} / \mathrm{side}$. (c) $* P<0.05$, main effects for food type (chow different from chocolate shake) and dose (I and $5 \mu \mathrm{g}$ different from vehicle). (e) $* * P<0.0 \mathrm{I}$, main effect of food type. (g) ** $P<0.01$, different from vehicle, when collapsed across the food-type factor (following a main effect of dose). Error bars depict one SEM.

Note that, overall, the number of bouts and total eating duration differed slightly between the chow and chocolateshake conditions for the CNQX group (main effect of food type for the number of bouts: $\mathrm{F}(1,5)=6.8, P=0.048$; for total eating duration: $\mathrm{F}(1,5)=25.2, P=0.004)$.

Finally, for latency to initiate the first feeding bout, there was a main effect of drug $(\mathrm{F}(1,10)=5.6, P=0.039)$ and dose $(\mathrm{F}(2,20)=5.6, P=0.01)$, but no effect of food type, and no interactions among the drug, dose, and food-type factors. The main effects of drug and dose were due to a tendency for AP-5 to increase feeding latency, regardless of food type. These effects on latency are shown in Supplementary Table S2.

\section{Microanalysis of Water Drinking}

For all experiments, there were main effects of food type for water intake (vmPFC $v$ s AIC experiment: $\mathrm{F}(1,13)=35.1$, $P<0.0001 ; \quad$ CNQX $v s$ AP-5 experiment: $\mathrm{F}(1,10)=25.7$, $P=0.0005$ ), number of drinking bouts (vmPFC vs AIC experiment: $\mathrm{F}(1,13)=16.3, P=0.001)$; $\mathrm{CNQX} v s \mathrm{AP}-5$ experiment: $\mathrm{F}(1,10)=27.3, \quad P=0.0004)$, total drinking duration (vmPFC vs AIC experiment: $\mathrm{F}(1,13)=40.9, P<0.0001$; CNQX vs AP-5 experiment: $\mathrm{F}(1,10)=68.4, P<0.0001)$, mean drinking bout duration (vmPFC vs AIC experiment: $\mathrm{F}(1,13)=22.7$, $P=0.0004$; CNQX $v s$ AP-5 experiment: $\mathrm{F}(1,10)=29.6$, $P=0.0003$ ), and latency to drink (vmPFC vs AIC experiment: 
a

vmPFC

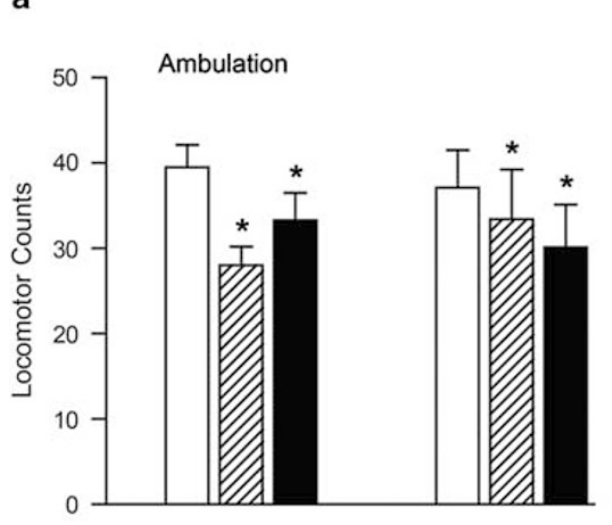

C

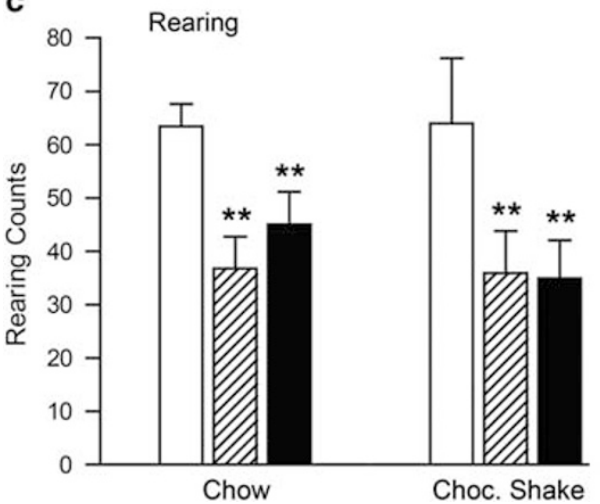

b

AIC

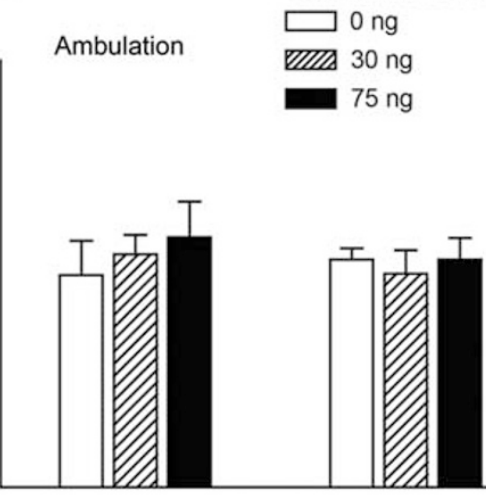

d

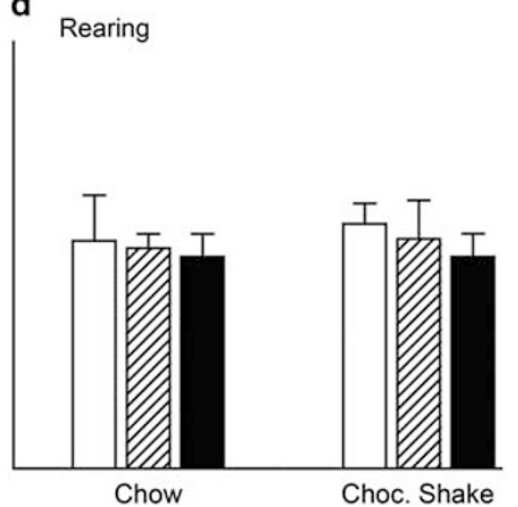

Figure 5 Ambulation and rearing behavior after muscimol infusions into the ventral medial prefrontal cortex ('vmPFC'; panels a and c) or agranular insular cortex ('AIC'; panels b and d). Muscimol doses for the bilateral injections are shown in ng/side. In panels (a) and (c), *P<0.05; *** $P<0.0$ l, different from vehicle, when collapsed across the food-type factor (main effect of dose). Error bars depict one SEM.

$\mathrm{F}(1,13)=8.5, \quad P=0.012 ; \quad \mathrm{CNQX} \quad$ vs AP-5 experiment: $\mathrm{F}(1,10)=21.1, P=0.001)$. These effects reflect the fact there was more overall water-drinking behavior in the chow condition compared with the liquid chocolate-shake condition in all the brain regions and with all the drugs. There were no significant interactions of food type with the brain region, drug, or dose factors. Note that, in the CNQX vs AP-5 experiment, rats of the AP-5 cohort spent more time drinking as a group, including with vehicle treatment. This effect was as reflected by main effects of drug (but not dose) for water intake $(\mathrm{F}(1,10)=54.6, \quad P<0.0001)$ and total drinking duration $(\mathrm{F}(1,10)=7.0, P=0.024)$.

Water intake and microstructure measures for the vmPFC vs AIC experiment are shown in Supplementary Table S1 and for the intra-vmPFC CNQX vs AP-5 experiment in Supplementary Table S3.

\section{Ambulation and Rearing}

As shown in Figure 5, there were distinct, site-specific effects of muscimol infusions upon ambulation and rearing in the vmPFC and AIC. For both ambulation and rearing, there were brain region $\times$ dose interactions (for ambulation: $\mathrm{F}(2,26)=3.7, P=0.038$; for rearing: $\mathrm{F}(2,26)=4.6, P=0.02)$. Further analyses with two-factor ANOVAs (food type $\times$ dose) within each brain region indicated that intra-vmPFC muscimol suppressed both locomotion and rearing (main effect of dose $(\mathrm{F}(2,14)=4.8, \quad P=0.026$ for locomotion; $\mathrm{F}(2,14)=9.6, P=0.0023$ for rears; Figure $5 \mathrm{a}$ and $\mathrm{c})$. In contrast, there were no effects of intra-AIG muscimol infusions on locomotor activity or rearing for either foodtype condition (Figure $5 \mathrm{~b}$ and $\mathrm{d}$ ). The suppression of rearing induced by intra-vmPFC muscimol was mimicked by intravmPFC CNQX but not AP-5 (drug $\times$ dose interaction: $\mathrm{F}(2,20)=5.8, P=0.01$; main effect of CNQX in the vmPFC: $\mathrm{F}(2,10)=4.6, P=0.039$; Supplementary Table S3).

Note that, for ambulation in the vmPFC vs AIC experiment, there was a main effect of brain region $(\mathrm{F}(1,13)=5.4, \quad P=0.037)$, reflecting the fact that overall ambulation levels were slightly greater in the vmPFC group vs the AIC group.

Results for all experiments and measures are summarized in tabular form in Supplementary Table S4.

\section{DISCUSSION}

Reversible inactivation of the AIC $v s$ vmPFC produced distinguishable effects on food intake and feeding microstructure across two distinct feeding conditions. Intra-AIC muscimol suppressed palatable feeding in ad libitum-maintained rats, as evidenced by a selective decrease in chocolate-shake intake and duration of time spent ingesting chocolate shake, but did not affect ingestion of standard chow in 
food-deprived rats. Neither ambulation nor rearing was altered by intra-AIC muscimol. In contrast, intra-vmPFC muscimol shifted the pattern of feeding behavior toward longer individual bouts, regardless of whether feeding was elicited by food deprivation or palatability, while slightly decreasing the output of exploratory-like responses (ambulation and rearing). This change in behavioral organization was insufficient to alter overall levels of chow or chocolateshake intake. The effects of intra-vmPFC muscimol were mimicked by local infusions of an AMPA-type glutamate receptor antagonist, but not an NMDA-type antagonist, indicating that modulation of feeding microstructure occurs through endogenous AMPA-receptor signaling in the vmPFC. Water intake and the microstructure of waterdrinking were not significantly changed by muscimolmediated inactivation of either site. Together, these results demonstrate a regional heterogeneity in the frontal control of feeding behavior: the AIC modulates palatability-driven feeding but is not necessary for hunger-driven intake of a less-appetizing food, while the vmPFC regulates the organization of feeding regardless of how the feeding is elicited.

The selective decrease in palatable feeding after AIC inactivation can be interpreted in the context of the rich convergence of gustatory, olfactory, somato-sensory, viscerosensory, and limbic input in this region (Kosar et al, 1986; Allen et al, 1991; Maffei et al, 2012). Units in the AIC and overlying dysgranular insular cortex integrate somatosensory information (including that arising from orofacial responses), and chemosensory information into a complex temporal code (Katz et al, 2001). In addition, neurons in these areas encode associative relationships between environmental cues and specific taste outcomes or tasteant availability (Saddoris et al, 2009; Samuelsen et al, 2012). Therefore, beyond its role in gustatory processing during the act of feeding, the AIC also performs computations relevant to cued food-approach behavior. Hence, it is interesting that, in the present study, AIC inactivation reduced chocolateshake intake and shortened the duration of this intake but affected neither the latency to initiate intake nor the number of bouts initiated. This pattern of results suggests that, in a free-feeding context, neural activity in the AIC is not necessary for food-directed approach. Instead, the present effects of AIC inactivation may have been due to impairment of chemosensory and/or oral somato-sensory perception during ingestion of the shake, which caused a corresponding change in food-reward valuation. Specifically, by changing taste characteristics, AIC inactivation may have rendered the chocolate shake less rewarding than expected. This interpretation is consistent with classic studies of sucrose-licking microstructure; less-concentrated (presumably less rewarding) sucrose solutions support shorter periods of sustained licking relative to more concentrated solutions (Davis and Smith, 1992). An alternative interpretation is that AIC inactivation caused a global motivational deficit instead of a specific impairment in gustatory perception or taste-reward valuation. Again, however, the lack of intra-AIC muscimol effects on measures related to food-directed approach argue against this interpretation. AIC inactivation also failed to affect hunger-driven chow intake, further demonstrating a lack of global motivational deficit, and indicating that putative AIC-based computations reflecting hungerinduced amplification of food reward are not necessary to support normal levels of chow intake in a state of energy deficit. The lack of effect on hunger-driven chow intake likely reflects the fact that AIC-based processing is only one of the several redundant pathways that conveys motivationally relevant information to 'feeding control columns' at lower levels of the neural axis during conditions of acute negative energy balance. In summary, the present data indicate that neural processing in the AIC is required for normal levels of palatable food intake but is not required for intake driven by energy deficit.

In contrast to inactivation of the AIC, vmPFC inactivation or AMPA receptor blockade did not alter the intake of chow or chocolate shake. Hence, despite the fact that palatability is encoded in the vmPFC (Jezzini et al, 2013), activity in this structure is not necessary to support normal levels of either palatability- or hunger-driven feeding. Instead, muscimolmediated vmPFC inactivation produced a significant increase in the duration of individual feeding bouts. This change in microstructure confirms previous observations from our laboratory (Mena et al, 2011) and was replicated by intravmPFC AMPA-receptor blockade. The underlying mechanism responsible for this change in behavioral organization is unknown, but several potential mechanisms come to mind. It is possible, for example, that the feeding-bout lengthening was the indirect consequence of a generalized motor deficit that diminished competing behaviors. Arguing against a global motor deficit, however, is the fact that intra-vmPFC muscimol altered neither feeding latency nor any parameter of water drinking. Similarly, because vmPFC inactivation changed neither total intake nor the number of feeding bouts initiated (although there was a non-significant trend toward fewer bouts), it is unlikely that this manipulation caused an overall change in the rewarding properties of food. In this regard, it is interesting to compare the effects of intra-vmPFC muscimol inactivation and $\mu$-opioid receptor $(\mu \mathrm{OR})$ stimulation. Intra-vmPFC $\mu \mathrm{OR}$ stimulation, which clearly amplifies food motivation as evidenced by elevated food-reinforced progressive-ratio responding (Mena et al, 2013), produces a microstructure pattern essentially the opposite of that seen here with muscimol or CNQX: marked hyperactivity, increased number of feeding bouts initiated, and decreased duration of individual feeding bouts (Mena et al, 2011). These marked dissimilarities between the microstructure changes incurred by intra-vmPFC muscimol (or CNQX) vs $\mu \mathrm{OR}$ stimulation indirectly suggests that the increase in mean feeding bout duration seen here was not due to an enhancement of food motivation.

A more likely explanation is that intra-vmPFC muscimol or CNQX altered temporal control over individual feeding bouts. Consummatory behavior consists of stereotyped movement patterns organized into bouts or clusters separated from one another by intervals ranging up to hundreds of seconds (Smith, 1998; Spector et al, 1998). Studies have suggested that frontal control over feeding microstructure occurs at the level of modulating the initiation and cessation of these clusters. For example, electrophysiological studies have identified units and ensembles in the PFC and orbitofrontal cortex showing sustained periods of excitation or inhibition time-locked to licking clusters or which predict the onset of licking clusters (Gutierrez et al, 2006; Petyko et al, 2009; Horst and Laubach, 2013). The present results suggest that vmPFC activity 
normally limits the amount of time spent in each feeding bout or cluster. This function could be achieved through the generation of a 'stop-signal', which halts consummatory motor patterns, or which codes a switch to an alternative behavioral set (eg, eating $\rightarrow$ environmental reconnaissance). Either mechanism would be consistent with the present observations that vmPFC inactivation lengthened feeding bouts and slightly suppressed exploratory-like behavior. It is tempting to speculate that vmPFC activity prevents the animal from becoming locked into long bouts of 'automatic' consummatory actions at the expense of adaptive, active engagement with the environment (eg, periodic assessment of possible new contingencies in the form of threats and/or richer sources of food). In a general sense, this hypothesis concurs with the established role of the vmPFC in restraining prepotent or disadvantageous responses, or previously extinguished repertoires, in order to align behavioral performance with current environmental contingencies (Narayanan et al, 2006; Rhodes and Killcross, 2007; Peters et al, 2009).

The fact that intra-vmPFC CNQX mimicked many of muscimol's behavioral effects suggests that AMPA transmission is physiologically relevant for vmPFC control of feeding microstructure. It is not clear why AMPA blockade influenced feeding microstructure while NMDA blockade did not. Doses of the NMDA antagonist, AP-5, used in the current study are behaviorally active in rat PFC, as evidenced by studies showing modulation of instrumental learning and drug place conditioning (Baldwin et al, 2000; Bishop et al, 2011; Tan et al, 2014). Both receptors are localized on pyramidal neurons and both contribute to the generation of postsynaptic EPSCs but with slight functional differences: the AMPA component is characterized by more rapid onset, while the NMDA component displays slower kinetics (Umemiya et al, 1999; Myme et al, 2003). These subtle but significant differences may impart distinguishable behavioral functions to these receptors. For example, partly because of its slower kinetic profile, intra-PFC NMDA signaling is hypothesized to have a crucial role in stabilizing delayrelated network activity in working-memory tasks (Wang et al, 2013). Moreover, PFC-localized NMDA receptors are required for learning-related plasticity (Baldwin et al, 2000; Barker and Warburton, 2008). AMPA receptors are also strongly implicated in cellular plasticity; however, the present data suggest that these receptors additionally have a selective role in transducing phasic signals that modulate ongoing appetitive behaviors in real time. Accordingly, an earlier study showed that activation of PFC unit spiking by lowfrequency stimulation of hippocampal efferent pathways to the vmPFC is mediated selectively AMPA-type receptors (Jay et al, 1992). This finding is consistent with the possibility that putative phasic, feeding-modulatory signals of limbic origin may impact PFC activity through AMPA receptor signaling. Further work is needed to assess these issues and to test the role of additional glutamate receptor subtypes in the control of feeding microstructure.

To summarize, the present findings reveal regional heterogeneity in the frontal control of hunger-driven $v s$ palatability-driven feeding in the rat. Regions of the AIC corresponding to gustatory cortex modulate palatable feeding in the ad libitum-fed state but are not necessary for hungerassociated intake of a less-palatable food. The vmPFC appears to influence the duration of individual feeding bouts regardless of how the feeding is elicited, possibly by coding a signal to terminate ongoing feeding bouts and switch to alternative behaviors. Regarding the clinical implications of these findings, an interesting possibility is that insular cortex has a role in amplifying food intake beyond homeostatic need when intake is driven by taste reward, whereas pathological changes in vmPFC function impair the ability to switch away from palatable-food binges once they have begun. More generally, the present findings provide an anatomical and mechanistic framework with which to interpret aberrant frontal activity in imaging studies of individuals with eating disorders, obesity, and other disorders of appetitive motivation.

\section{FUNDING AND DISCLOSURE}

This work was funded by National Institute of Mental Health R01 grant MH074723 (PI on this award: Dr Brian A Baldo). Dr Jesus Mena was supported by Predoctoral NRSA fellowship F31 DA 028104. The authors declare no conflict of interest.

\section{ACKNOWLEDGMENTS}

We thank Mr Ryan Selleck for assistance with creating the data figures and Dr Vaishali Bakshi for helpful comments on the manuscript.

\section{REFERENCES}

Allen GV, Saper CB, Hurley KM, Cechetto DF (1991). Organization of visceral and limbic connections in the insular cortex of the rat. J Comp Neurol 311: 1-16.

Baldwin AE, Holahan MR, Sadeghian K, Kelley AE (2000). $\mathrm{N}$-methyl-D-aspartate receptor-dependent plasticity within a distributed corticostriatal network mediates appetitive instrumental learning. Behav Neurosci 114: 84-98.

Barker GR, Warburton EC (2008). NMDA receptor plasticity in the perirhinal and prefrontal cortices is crucial for the acquisition of long-term object-in-place associative memory. J Neurosci 28: 2837-2844.

Basso AM, Kelley AE (1999). Feeding induced by GABA(A) receptor stimulation within the nucleus accumbens shell: regional mapping and characterization of macronutrient and taste preference. Behav Neurosci 113: 324-336.

Berridge KC (1991). Modulation of taste affect by hunger, caloric satiety, and sensory-specific satiety in the rat. Appetite 16: 103-120.

Berthoud HR (2011). Metabolic and hedonic drives in the neural control of appetite: who is the boss? Curr Opin Neurobiol 21: 888-896.

Bishop SF, Lauzon NM, Bechard M, Gholizadeh S, Laviolette SR (2011). NMDA receptor hypofunction in the prelimbic cortex increases sensitivity to the rewarding properties of opiates via dopaminergic and amygdalar substrates. Cereb Cortex 21: 68-80.

Cabanac M, Duclaux R (1973). Olfactory-gustatory alliesthesia and food intake in humans. J Physiol 66: 113-135.

Cameron JD, Goldfield GS, Finlayson G, Blundell JE, Doucet E (2014). Fasting for $24 \mathrm{~h}$ heightens reward from food and foodrelated cues. PLoS One 9: e85970.

Davis JD, Smith GP (1992). Analysis of the microstructure of the rhythmic tongue movements of rats ingesting maltose and sucrose solutions. Behav Neurosci 106: 217-228. 
Floyd NS, Price JL, Ferry AT, Keay KA, Bandler R (2001). Orbitomedial prefrontal cortical projections to hypothalamus in the rat. J Comp Neurol 432: 307-328.

Gabbott PL, Warner TA, Jays PR, Bacon SJ (2003). Areal and synaptic interconnectivity of prelimbic (area 32), infralimbic (area 25) and insular cortices in the rat. Brain Res 993: 59-71.

Goldstone AP, Prechtl de Hernandez CG, Beaver JD, Muhammed $\mathrm{K}$, Croese C, Bell G et al (2009). Fasting biases brain reward systems towards high-calorie foods. Eur J Neurosci 30: 1625-1635.

Gutierrez R, Carmena JM, Nicolelis MA, Simon SA (2006). Orbitofrontal ensemble activity monitors licking and distinguishes among natural rewards. J Neurophysiol 95: 119-133.

Horst NK, Laubach M (2013). Reward-related activity in the medial prefrontal cortex is driven by consumption. Front Neurosci 7: 56.

Hurley KM, Herbert H, Moga MM, Saper CB (1991). Efferent projections of the infralimbic cortex of the rat. J Comp Neurol 308: 249-276.

Jay TM, Thierry AM, Wiklund L, Glowinski J (1992). Excitatory amino acid pathway from the hippocampus to the prefrontal cortex. contribution of AMPA receptors in hippocampoprefrontal cortex transmission. Eur J Neurosci 4: 1285-1295.

Jezzini A, Mazzucato L, La Camera G, Fontanini A (2013). Processing of hedonic and chemosensory features of taste in medial prefrontal and insular networks. J Neurosci 33: 18966-18978.

Katz DB, Simon SA, Nicolelis MA (2001). Dynamic and multimodal responses of gustatory cortical neurons in awake rats. J Neurosci 21: $4478-4489$.

Killgore WD, Young AD, Femia LA, Bogorodzki P, Rogowska J, Yurgelun-Todd DA (2003). Cortical and limbic activation during viewing of high- versus low-calorie foods. Neuroimage 19: 1381-1394.

Kosar E, Grill HJ, Norgren R (1986). Gustatory cortex in the rat. II. Thalamocortical projections. Brain Res 379: 342-352.

Land BB, Narayanan NS, Liu RJ, Gianessi CA, Brayton CE, Grimaldi DM et al (2014). Medial prefrontal D1 dopamine neurons control food intake. Nat Neurosci 17: 248-253.

Maffei A, Haley M, Fontanini A (2012). Neural processing of gustatory information in insular circuits. Curr Opin Neurobiol 22: 709-716.

Martin LE, Holsen LM, Chambers RJ, Bruce AS, Brooks WM, Zarcone JR et al (2010). Neural mechanisms associated with food motivation in obese and healthy weight adults. Obesity 18: 254-260.

Mena JD, Sadeghian K, Baldo BA (2011). Induction of hyperphagia and carbohydrate intake by mu-opioid receptor stimulation in circumscribed regions of frontal cortex. J Neurosci 31: 3249-3260.

Mena JD, Selleck RA, Baldo BA (2013). Mu-opioid stimulation in rat prefrontal cortex engages hypothalamic orexin/hypocretincontaining neurons, and reveals dissociable roles of nucleus accumbens and hypothalamus in cortically driven feeding. J Neurosci 33: 18540-18552.

Morris MJ, Beilharz JE, Maniam J, Reichelt AC, Westbrook RF (2014). Why is obesity such a problem in the 21 st century? The intersection of palatable food, cues and reward pathways, stress, and cognition. Neurosci Biobehav Rev (doi:10.1016/jneurbiorev. 2014.12.002; e-pub ahead of print).

Myme CI, Sugino K, Turrigiano GG, Nelson SB (2003). The NMDA-to-AMPA ratio at synapses onto layer $2 / 3$ pyramidal neurons is conserved across prefrontal and visual cortices. J Neurophysiol 90: 771-779.

Narayanan NS, Horst NK, Laubach M (2006). Reversible inactivations of rat medial prefrontal cortex impair the ability to wait for a stimulus. Neuroscience 139: 865-876.

Newman S, Pascal L, Sadeghian K, Baldo BA (2013). Sweetened-fat intake sensitizes gamma-aminobutyric acid-mediated feeding responses elicited from the nucleus accumbens shell. Biol Psychiatry 73: 843-850.
Perry ML, Baldo BA, Andrzejewski ME, Kelley AE (2009). Muscarinic receptor antagonism causes a functional alteration in nucleus accumbens mu-opiate-mediated feeding behavior. Behav Brain Res 197: 225-229.

Peters J, Kalivas PW, Quirk GJ (2009). Extinction circuits for fear and addiction overlap in prefrontal cortex. Learn Mem 16: 279-288.

Petrovich GD, Holland PC, Gallagher M (2005). Amygdalar and prefrontal pathways to the lateral hypothalamus are activated by a learned cue that stimulates eating. J Neurosci 25: 8295-8302.

Petrovich GD, Risold PY, Swanson LW (1996). Organization of projections from the basomedial nucleus of the amygdala: a PHAL study in the rat. J Comp Neurol 374: 387-420.

Petyko Z, Toth A, Szabo I, Galosi R, Lenard L (2009). Neuronal activity in rat medial prefrontal cortex during sucrose solution intake. Neuroreport 20: 1235-1239.

Pritchard TC, Hamilton RB, Morse JR, Norgren R (1986). Projections of thalamic gustatory and lingual areas in the monkey, Macaca fascicularis. J Comp Neurol 244: 213-228.

Reynolds SM, Zahm DS (2005). Specificity in the projections of prefrontal and insular cortex to ventral striatopallidum and the extended amygdala. J Neurosci 25: 11757-11767.

Rhodes SE, Killcross AS (2007). Lesions of rat infralimbic cortex enhance renewal of extinguished appetitive Pavlovian responding. Eur J Neurosci 25: 2498-2503.

Rolls ET (2012). Taste, olfactory and food texture reward processing in the brain and the control of appetite. Proc Nutr Soc 71: 488-501.

Saddoris MP, Holland PC, Gallagher M (2009). Associatively learned representations of taste outcomes activate tasteencoding neural ensembles in gustatory cortex. J Neurosci 29: 15386-15396.

Samuelsen CL, Gardner MP, Fontanini A (2012). Effects of cuetriggered expectation on cortical processing of taste. Neuron 74: $410-422$.

Shi CJ, Cassell MD (1998). Cortical, thalamic, and amygdaloid connections of the anterior and posterior insular cortices. J Comp Neurol 399: 440-468.

Small DM (2010). Taste representation in the human insula. Brain Struct Funct 214: 551-561.

Small DM, Zatorre RJ, Dagher A, Evans AC, Jones-Gotman M (2001). Changes in brain activity related to eating chocolate: from pleasure to aversion. Brain 124: 1720-1733.

Smith GP (1998). Satiation: From Gut to Brain. Oxford University Press: New York, NY, USA, 291pp.

Spector AC, Klumpp PA, Kaplan JM (1998). Analytical issues in the evaluation of food deprivation and sucrose concentration effects on the microstructure of licking behavior in the rat. Behav Neurosci 112: 678-694.

Tan H, Rosen LG, Ng GA, Rushlow WJ, Laviolette SR, Addiction Research Group (2014). NMDA receptor blockade in the prelimbic cortex activates the mesolimbic system and dopamine-dependent opiate reward signaling. Psychopharmacology (Berl) 231: 4669-4679.

Umemiya M, Senda M, Murphy TH (1999). Behaviour of NMDA and AMPA receptor-mediated miniature EPSCs at rat cortical neuron synapses identified by calcium imaging. J Physiol 521: 113-122.

Vertes RP (2004). Differential projections of the infralimbic and prelimbic cortex in the rat. Synapse 51: 32-58.

Wang M, Yang Y, Wang CJ, Gamo NJ, Jin LE, Mazer JA et al (2013). NMDA receptors subserve persistent neuronal firing during working memory in dorsolateral prefrontal cortex. Neuron 77: 736-749.

Yaxley S, Rolls ET, Sienkiewicz ZJ (1990). Gustatory responses of single neurons in the insula of the macaque monkey. J Neurophysiol 63: 689-700. 